\title{
Aetiology for gastro intestinal reflux disease
}

\section{Weeerawardena WAK}

Department of Surgery - Teaching Hospital Anuradhapura

\begin{abstract}
\section{Introduction}

Gastro intestinal reflux disease(GORD) is common. The incidence is rising in the world and in Sri Lanka. Food habits, behavioural pattern are considered to be of concerned about aetiology.
\end{abstract}

\section{Objective}

To identify the risk factors for GORD to make a plan for reducing the incidence o0f symptoms.

\section{Materials and methods}

Reviewed 23 journal articles published during last 15 years of duration. Key words of GORD, aetiology, risk factors were used. Different possible aetiological factors were identified and their relationship to GORD was assessed.

\section{Results}

Gastric acid, tobacco smocking, table salt use, dietary factors, alcohol, physical activity, Body mass index, obesity, ethnicity, gender, posture, genetic contribution, medication effect, role of Helicobactor pylori, effect of naso gastric tubes, hiatus hernia, pregnancy, other cardiac diseases were identified for considering as aetiology. Special variety of infantile GORD was also selected.

\section{Discusion and conclusion}

Exact aetiology of GORD is largely unknown. However this review has detected several significant associations with GORD. Knowledge of those association factors are useful in the management of the symptoms. Overweight, obesity, tobacco smocking, alcohol, high salt intake, fibre intake, physical exercise are some of the association factors. People have capacity to alter those factors with view to reduce the incidence of GORD

\section{Introduction}

GORD is common. The incidence is rising in the world and in Sri Lanka. Food habits, behavioural pattern are considered to be of concerned about aetiology. The therapeutic options are wide. However treatment may include long term PPI and laparotomy or thoracotomy with variable results. It is the time to investigate for the cause GORD. Adherent to preventive steps will abolish

the complications of long term medication and extensive surgeries and the expenses involved. I have reviewed index journal literature published during last 15 years duration.

Gastro-oesophageal reflux disease is a condition with the reflux of gastric contents into the oesophagus. This reflux provokes symptoms or complications and impairs quality of life. Typical symptoms of gastro-oesophageal reflux disease are ,

1. heartburn

2. regurgitation

However gastro-oesophageal reflux disease has also been related to extra-oesophageal manifestations, such as asthma, chronic cough and laryngitis. The pathogenesis of gastrooesophageal reflux disease is multifactorial. This involve transient lower oesophageal sphincter relaxations and other lower oesophageal sphincter pressure abnormalities. Reflux of acid, bile, pepsin and pancreatic enzymes cause oesophageal mucosal injury(1). 
Other factors contributing to the

pathophysiology of gastro-oesophageal reflux

disease include,

1.hiatal hernia,

2. impaired oesophageal clearance,

3.delayed gastric emptying

4. impaired mucosal defensive factors.

Hiatal hernia contributes to gastro-oesophageal reflux disease by promoting lower oesophageal sphincter dysfunction. Impaired oesophageal clearance is responsible for prolonged acid exposure of the mucosa. Delayed gastric emptying, resulting in gastric distension, can significantly increase the rate of transient lower oesophageal sphincter relaxations, contributing to postprandial gastro-oesophageal reflux disease $(1,2)$.

\section{Method}

Pubmed search were carried out to find out the aetiological factors for GORD. 22 journal articles were reviewed published during last 15 years. Key words of GORD, aetiology, risk factors were used. Different aetiological factors were identified and their relationship to GORD was assessed.

\section{Results}

Gastric acid, tobacco smocking, table salt use, dietary factors, alcohol, physical activity, Body mass index, obesity, ethnicity, gender, posture, genetic contribution, medication effect, role of Helicobactor pylori, effect of naso gastric tubes, hiatus hernia, pregnancy, other cardiac diseases were identified for considering as aetiology. Special variety of infantile GORD was also selected.

\section{Gastric acid}

Aetiology for gastro intestinal reflux disease

The main diseases associated with dyspepsia are peptic ulcer disease, gastro-oesophageal reflux disease and non-ulcer dyspepsia. Increased gastric acid secretion is a characteristic of most duodenal ulcer patients and of a small minority of non-ulcer dyspepsia and GORD patients. Although acid secretion is normal in most GORD patients, the condition is mainly the result of excess exposure of the distal oesophagus to acid refluxing from the stomach. Increased mucosal sensitivity to acid is the aetiology of dyspeptic symptoms in the majority of patients with peptic ulcer disease and GORD, and in a minority of nonulcer dyspepsia subjects. Gastric acid, therefore, plays an important role in both the aetiology of dyspeptic diseases and in the aetiology of dyspeptic symptoms (3)

\section{Tobacco smocking, alcohol and table salt}

The aetiology of gastro-oesophageal reflux is largely unknown. In Norway, a case control study within the two public health surveys, including 3153 individuals reported severe heartburn or regurgitation during the last 12 months were defined as case. They included 40210 people without reflux symptoms to constitut the control group. There was a significant dose response association between tobacco smoking and reflux symptoms. Among people who had smoked daily for more than 20 years the odds ratio was 1.7 (95\% confidence interval 1.5 to 1.9 ) compared with non-smokers. $(4,5)$ Another large, population based study provides that tobacco smoking causes symptomatic GORD.(11)

In two large population base studies, alcohol was not identified as a risk factor(4)(11). However total of 7124 subjects were interviewed as part of the German National Health Interview and Examination Survey, a representative sample of the general adult population. They found that smoking and the frequent consumption of spirits are risk factors for GORD.(5)GORD shows a positive association with table salt intake. The odds ratio for reflux was 1.7 (95\% CI 
1.4 to 2.0) among those who always used extra table salt compared with those who never did so.(4) Another large, population based study shows that table salt intake seem to be risk factors for gastro-oesophageal reflux symptoms(11).

\section{Dietary factors}

A team used PubMed and Ovid to perform a search of the literature published between 1975 and 2004 using the key words heartburn, GERD(Gastro esophageal reflux disease), smoking, alcohol, obesity, weight loss, caffeine or coffee, citrus, chocolate, spicy food, head of bed elevation, and late-evening meal. Although there was physiologic evidence that exposure to tobacco, alcohol, chocolate, and high-fat meals decreases lower esophageal sphincter pressure, there was no published evidence of the efficacy of dietary measures. Neither tobacco nor alcohol cessation was associated with improvement in esophageal $\mathrm{pH}$ profiles or symptoms (9).

In two large population base studies, alcohol was not identified as a risk factor $(4,11)$. However total of 7124 subjects were interviewed as part of the German National Health Interview and Examination Survey, a representative sample of the general adult population. They found that smoking and the frequent consumption of spirits are risk factors for GORD.(5)

A team systematically reviewed the pathogenetic link between overweight/obesity, dietary habits, physical activity and GERD, and the beneficial effect of specific recommended changes, by means of the available literature from the 1999 to 2009.(10). The role of dietary behavior, mainly in terms of specific dietary components, remains controversial. Mild routine physical activity in association with diet modifications, i.e. a diet rich in fiber and low in fat, is advisable in preventing reflux symptoms $(10,4)$. Physical activity and the consumption of fruits seemed to have some protective effect and sweets, or white bread are risk factors for GORD (5)
A large population based study provides firm evidence that dietary fibres may protect against reflux.(11)(4).Coffee, and tea do not seem to be risk factors for reflux(4)(11).

\section{Overweight and obesity.}

A total of 7124 subjects were interviewed as part of the German National Health Interview and Examination Survey, a representative sample of the general adult population. They found an association among those with reflux symptoms who were overweight and obese (odds ratio: 1.8, 95\% confidence interval: $1.5-2.2$; odds ratio: 2.6 , 95\% confidence interval: $2.2-3.2$ ), respectively.

A study demonstrated a clear and dosedependent association between increasing degrees of overweight and gastro-oesophageal reflux. The mechanisms by which obesity causes reflux are unknown though there is some limited data to suggest that hiatal hernia may be the causal link between obesity and reflux. There are some evidence that obesity is clearly a stronger risk factor among women than among men, and that the relation between overweight and reflux is substantially augmented by postmenopausal hormone therapy(6). Weight-reduction seems to reduce the risk of symptomatic GORD , indicating that such strategy might be a useful tool in the treatment of reflux. (6)(9).

In 2000, a team used a supplemental questionnaire to determine the frequency, severity, and duration of symptoms of gastroesophageal reflux disease among randomly selected participants in a Study. Of 10,545 women who completed the questionnaire (response rate, 86 percent), 2310 (22 percent) reported having symptoms at least once a week, and 3419 (55 percent of those who had any symptoms) described their symptoms as moderate in severity.(7) Body mass index (BMI) is associated with symptoms of gastroesophageal reflux disease in both normal-weight and overweight women. Even moderate weight gain among persons of normal weight may cause or exacerbate symptoms of reflux (7). 
A study was done to systematically review the pathogenetic link between overweight/obesity, dietary habits, physical activity and GORD, and the beneficial effect of specific recommended changes, by means of the available literature from the 1999 to 2009(10).This team concluded that, being obese/overweight and GORD-specific symptoms and endoscopic features are related, and weight loss significantly improves GORD clinical-endoscopic manifestations (10)

\section{Physical activity}

A large, population based study provides firm evidence that physical exercise may protect against reflux $(11,4,5)$.

\section{Ethnicity and gender.}

There was a consistent association between abdominal diameter (independent of BMI) and reflux-type symptoms in the white population, but no consistent associations in the black population or Asians. The BMI association was also strongest among the white population. These findings, combined with the increased prevalence of abdominal obesity in male subjects, suggest that an increased obesity may disproportionately increase GORD-type symptoms in the white population and in male subjects. (8)

\section{Posture}

Head of bed elevation and left lateral decubitus position improved the overall time that the esophageal $\mathrm{pH}$ was less than 4.0. Weight loss and head of bed elevation are effective lifestyle interventions for GERD(9)

\section{Nasogastric tubes}

A prospective randomized case-control study with 15 consenting patients demonstrated that patients undergoing elective laparotomy with routine nasogastric tube placement have significant gastroesophageal reflux in the perioperative period and shows reduced ability to clear refluxed acid from the distal esophagus. Due to the associated risk of postoperative pulmonary complications, they recommend that nasogastric intubation be performed on a selective rather than routine basis.(12).

\section{Gastro oesophageal reflux in infants}

According to epidemiological data, the role of gastro-oesophageal reflux in the aetiology of this life threatening events is unclear.The incident of sudden infant death is decreased in the supine versus the prone sleeping position. On the contrary, gastro-oesophageal reflux is more pronounced in the supine than in the prone position, both in infants and in older children, both in infants with physiological and with pathological reflux. In the supine position, infants do sleep shorter, have more rapid-eye-movement sleep and have more arousals than in the prone position. It is thought that in the majority of infants, gastro-oesophageal reflux stimulates arousals and thus may well be considered as "protective" for rather than "provoking" sudden infant death. However the authors concluded that this hypothesis needs to be validated.(13).

\section{Genetic contribution}

A total of 4480 unselected twin pairs, identified from a national volunteer twin register, were asked to complete a validated symptom questionnaire about GORD in Glagow ,United Kingdom. GORD was defined as symptoms of heartburn or acid regurgitation at least weekly during the past year (14). They detected that there is a substantial genetic contribution to the aetiology of GORD.

\section{Relation to Helicobactor pylori}

Epidemiological studies demonstrate a negative association between Helicobacter pylori infection and gastro-oesophageal reflux disease and its complications. This might represent a protective effect for GORD as the tendency for $\mathrm{H}$. pylori infection lower the gastric acid secretion with advancing age. 
However, studies of the effect of $\mathrm{H}$. pylori eradication on GORD have failed to show any worsening of GORD symptoms. Helicobacter pylori infection improves the control of gastric acidity by proton-pump inhibitors and this produces a small advantage in clinical control of reflux disease. The infection prevents rebound acid hypersecretion occurring when protonpump inhibitor therapy is discontinued. However, concerns have been expressed that the body gastritis induced by proton-pump inhibitor therapy in $\mathrm{H}$. pylori-infected subjects might increase the risk of gastric cancer. At present, it is unclear whether H. pylori should be eradicated in gastro-oesophageal reflux disease patients.(15)

\section{Hiatus hernia}

Hiatal hernia, in combination with other reflux conditions and symptoms, was associated strongly with the risk of esophageal adenocarcinoma. These associations were more modest for gastric cardia adenocarcinomas. A significant and positive association between body size and history of hiatal hernia/reflux symptoms also was observed.(17)

\section{Medications}

A multicenter questionnaire survey concluded that though larger cohort is required for further study, the combination of calcium channel blo ckers and warfarin is an independent risk factor for GORD(18).

Oral bronchodilators are known to worsen GORD and may lead to vicious cycle when gastr oeso pha geal reflux causes bronchospasm. The effect of in haled bronchodilators on gastroeso phageal refl ux is unknown. Patients with gast roeso pha geal reflux disease, who require bron chodilator ther apy for obstructive lung disease, have less re flux with inhaled albuterol (22)
People experiencing frequent GOR D symptoms have markedly increased risks of oesophageal adenocarcinoma and gastro oesophageal junctional adeno carcinoma, and this effect may be greater amongst smokers. Use of aspirin and NSAIDs, but not acid suppressants, significantly reduced the risks of oesophageal cancers associated with GORD(22)

Naproxen did not induce reflux in normal subjects, although reflux did increase in some subjects

Individuals with long-standing GERD are at increased risk of esophageal adenocarcinoma, whether or not the symptoms are treated with $\mathrm{H} 2$ blockers or antacids(16)

\section{Other diseases}

A multicenter questionnaire survey demonstra ted that among traditional cardiovascular risk factors, AF was an independent risk factor for GERD. A large cohort study to assess the pote ntial relationship between GERD and AF is warranted. (19)

\section{Pregnancy}

Heartburn is a common symptom in pregnancy, affecting about two-thirds of pregnant women. The aetiology of GORD in pregnancy is multifactorial. Reduced lower basal gastrooesophageal sphi ncter pressure, increased intragastric pressure, delayed intestinal transit time and duodenogastric reflux have been found in pregnant women with heartburn, all factors which dispose to increased gastro-oesophageal reflux. Gastric emptying is apparently normal during pregnancy, but delayed during delivery. Therapy involves lifestyle modification and nonsystemic medication as the initial choices. $\mathrm{H}$ 2-antagonists should only be used in severe and refractory cases(20) 


\section{Discussion}

Exact aetiology of GORD is largely unknown. However this review has detected several significant association factors with GORD. Knowledge of those association factors is useful in the management of the symptoms. Overweight, obesity, tobacco smocking, alcohol, high salt intake, fibre intake, physical exercise are some of the association factors. People have capacity to alter those factors with view to reduce the incidence of GORD. It was also useful to review the association of the symptoms with tea and coffee. The relationship with the medications is valuable for the clinicians in their practice. The association of GORD with the nasogastric tube and posture need to be considered during inpatient management. The association of GORD with genetic changes, ethnicity and gender are useful in future research $(4,5,11)$.

Transient loosening of the lower oesophageal sphincter tone is suspected to be the mechanism of reflux. The angle between stomach and oesophagus, intra abdominal pressure over the intra peritoneal part of oesophagus are also play a role in preventing reflux. Those associated triggering factors for reflux are expected to alter the defense mechanism of anti reflux process. The anti reflux medication and surgical procedures are aiming at strengthening of those lower oesophageal sphincter tone and normalizing the angle between stomach and oesophagus. There is a failure rate of those surgical procedures and medical therapy and the therapeutic cost is very high to the community(2).

Once the symptoms star, the investigation with upper gastro intestinal endoscopy, Ba swallow, 24 hours ambulant lower oesophageal $\mathrm{pH}$ measurement with calculation of DeMeester score are useful. Considering about the cost of those investigations it is necessary to adhere to a preventing steps of the disease. Simple change of diet and behaviour will reduce the symptoms and consequences. Patient education and induction of exercise based weight reduction programs are expected to be favourable.

\section{Conclusion}

With the available data a preventive program can be arranged to reduce the incidence of GORD at North Central Province of Sri Lanka. Simple heath education may prevent sinister complications of GORD.

\section{References}

1) F De Giorgi, M Palmiero, I Esposito, F Mosca, R Cuomo. Pathophysiology of gastro-oesophageal reflux disease . Acta Otorhinolaryngol Ital. 2006 26(5): 241-246.

2)John Bancewicz. The oesophagus. In Baily and Love s Short Text Book of Surgery. RCG Russell, NS W illiams, CJK Bulstrode . 23 rd Edition, Arnold 2000 .863-871.

3)McColl KE. Role of gastric acid in the aetiology of dyspeptic disease and dyspepsia. Baillieres Clin Gastroenterol. 1998 ;12(3):489502.

4)Nilsson M, Johnsen R, Ye W, Hveem K, Lagergren J.Lifestyle related risk factors in the aetiology of gastro-oesophageal reflux.Gut. $2004 ; 53(12): 1730-5$.

5)Nocon M, Labenz J, Willich SN. Lifestyle factors and symptoms of gastro-oesophageal reflux -- a population-based study.Aliment Pharmacol Ther. 2006 1;23(1):169-74.

6)Nilsson M, Lagergren J. The relation between body mass and gastro-oesophageal reflux.Best Pract Res Clin Gastroenterol. 2004 Dec;18(6):1117-23.

7)Jacobson BC, Somers SC, Fuchs CS, Kelly CP, Camargo CA Jr. Body-mass index and symptoms of gastroesophageal reflux in women.N Engl J Med. 2006 1;354(22):2340-8.

8) Corley DA, Kubo A, Zhao W.Gut.Abdominal obesity, ethnicity and gastro-oesophageal reflux symptoms 2007 ;56(6):756-62. Epub 2006 Oct 17. 
(9)Review Kaltenbach T, Crockett S, Gerson LB.Arch Intern Med. Are lifestyle measures effective in patients with gastroesophageal reflux disease? An evidence-based

approach.2006;166(9):965-71.

10) Festi D, Scaioli E, Baldi F, Vestito A, Pasqui F, Di Biase AR, Colecchia A Body weight, lifestyle, dietary habits and gastroesophageal reflux disease World J Gastroenterol. 2009 14;15(14):1690-701.

11)M Nilsson,R Johnsen, $W$ Ye,K Hveem,J Lagergren . Lifestyle related risk factors in the aetiology of gastro-oesophageal reflux Gut. 2004; 53(12): 1730-1735.

12)Manning BJ, Winter DC, McGreal G, Kirwan WO, Redmond HPNasogastric intubation causes gastroesophageal reflux in patients undergoing elective laparotomy. Surgery. 2001;130(5):78891.

13)Vandenplas Y, Hauser B. Gastro-oesophageal reflux, sleep pattern, apparent life threatening event and sudden infant death. The point of view of a gastro-enterologist. Eur J Pediatr. 2000;159(10):726-9.

14) Mohammed I, Cherkas LF, Riley SA, Spector TD, Trudgill NJ. Genetic influences in gastrooesophageal reflux disease: a twin study. Gut. 2003;52(8):1085-9.

15) Delaney B, McColl K.Review article: Helicobacter pylori and gastro-oesophageal reflux disease. Aliment Pharmacol Ther. 2005 Aug;22 Suppl 1:32-40.

16) Farrow DC, Vaughan TL, Sweeney $C$, Gammon MD, Chow WH, Risch HA, Stanford JL, Hansten PD, Mayne ST, Schoenberg JB, Rotterdam H, Ahsan H, West AB, Dubrow R, Fraumeni JF Jr, Blot WJ.

Gastroesophageal reflux disease, use of $\mathrm{H} 2$ receptor antagonists, and risk of esophageal and gastric cancer. Cancer Causes Control. 2000 Mar;11(3):231-8.
Aetiology for gastro intestinal reflux disease

17) Wu AH, Tseng CC, Bernstein L. Hiatal hernia, reflux symptoms, body size, and risk of esophageal and gastric adenocarcinoma. . Cancer. 2003 1;98(5):940-8.

18)Nakaji G, Fujihara M, Fukata $M$, Yasuda $S$, Odashiro K, Maruyama T, Akashi K. Influence of common cardiac drugs on gastroesophageal reflux disease: multicenter questionnaire survey. Int J Clin Pharmacol Ther. 2011;49(9):555-62.

19) Shimazu H, Nakaji G, Fukata M, Odashiro K, Maruyama T, Akashi K; Fukuoka F-Scale Trial Group Relationship between atrial fibrillation and gastroesophageal reflux disease: a multicenter questionnaire survey. Cardiology. 2011;119(4):217-23

20)Okholm M, Jensen SM. [Gatroesophageal reflux in pregnant women] [Article in Danish] . Ugeskr Laeger. 1995 27;157(13):1835-8.

21)Ruzkowski CJ, Sanowski RA, Austin J, Rohwedder JJ, Waring JP. The effects of inhaled albuterol and oral theophylline on gastroesophageal reflux in patients with gastroesophageal reflux disease and obstructive lung disease. Arch Intern Med.

1992;152(4):783-5.

22)Pandeya N, Webb PM, Sadeghi S, Green AC, Whiteman DC; Australian Cancer Study.Gastrooesophageal reflux symptoms and the risks of oesophageal cancer: are the effects modified by smoking, NSAIDs or acid suppressants? Gut. 2010;59(1):31-8.

(23) Scheiman JM, Patel PM, Henson EK, Nostrant TT. Effect of naproxen on gastroesophageal reflux and esophageal function: a randomized, double-blind, placebocontrolled study. Am J Gastroenterol. 1995;90(5):754-7. 